\title{
ZEROS OF ENTIRE FUNCTIONS
}

BY

HANS-J. RUNCKEL

Introduction. This paper is the outgrowth of the author's Ph.D. thesis written under the supervision of Professor A. Peyerimhoff, whose paper on zeros of power series [4] has been the starting point.

$\$ 1$ contains some lemmas due to A. Peyerimhoff, which are needed in $\$ 2$.

In all theorems (with the exception of Theorem 4 only) of the remaining part of this paper, real entire functions $f(z)$ of finite order with infinitely many real and finitely many complex $\left({ }^{1}\right)$ zeros are taken as starting point.

Then in $\S 2$ functions of the form $g(z)=A(z) f(z)+B(z) f^{\prime}(z)$ are considered where $A(z)$ and $B(z)$ are real polynomials. In Theorem 1 upper bounds for the number of zeros of $g(z)$ in the complex plane with the exception of certain real intervals are obtained. In certain instances the exact number of zeros is obtained. At the end of $\$ 2$ examples are given which show that the results of Theorem 1 are best possible. Questions of this kind have been considered by Laguerre and Borel especially [1], [2] for the particular case $g(z)=f^{\prime}(z)$. One of the results of [4] deals with functions $g(z)=\alpha f(z)+z f^{\prime}(z)$ where $\alpha$ is real and $f(z)$ of order $<1$. The method of proof of this result has been generalized in the present paper.

In $\$ 3$ a couple of theorems $(2,3,5,6)$ which are derived from Theorem 1 are partly slight generalizations of known theorems, especially of Laguerre [2], [3], [5], [6]. These theorems deal with questions of the following kind. If one has some information on the zeros of the entire function $f(z)=\sum a_{n} z^{n}$, one wants to gain information on the zeros of $F(z)=\sum a_{n} G(n) z^{n}$, where $G(z)$ is an entire function of a certain type.

For Theorem 4, which is known already, a short proof is given here which is independent of Theorem 1. From this theorem follows immediately the wellknown fact that the Besselfunctions of real order $>-1$ have real zeros only.

From Theorem 6 Hurwitz's Theorem on the complex zeros of the Besselfunctions of real order $<-1$ follows as a special case.

In Theorem 9 functions $g(z)=\alpha f(z)+z^{l} f^{\prime}(z)$ are considered, where $\alpha$ is real, $l$ odd, and $f(z)$ is a real canonical product of finite genus. Then the exact number of complex zeros of $g(z)$ is obtained. Especially we find that for $\alpha>0$ and $l$ odd the functions $\alpha \sin z+z^{l} \cos z$ and $\alpha \cos z-z^{l} \sin z$ have exactly $l-1$ complex zeros.

1. In the proof of Lemma 1 we will need some elementary properties of difference quotients. In the following we suppose that $f(z)$ is a holomorphic function.

Received by the editors August 22, 1968 and, in revised form, February 3, 1969.

$\left.{ }^{1}\right)$ Here "complex" means "nonreal". 
Difference quotients which are undefined shall be defined by the corresponding differential quotients.

Let

$$
\Delta_{z_{1}} f(z)=\frac{f(z)-f\left(z_{1}\right)}{z-z_{1}}
$$

and recursively we define

$$
\Delta_{z_{l} \ldots z_{1}} f(z)=\Delta_{z_{l}}\left(\Delta_{z_{l}-1 \ldots z_{1}} f(z)\right) .
$$

This operation on functions $f(z)$ is homogeneous and linear, and for products $f(z)=g(z) \cdot h(z)$ we have $\Delta_{z_{1}}(g(z) h(z))=g\left(z_{1}\right) \Delta_{z_{1}} h(z)+h(z) \Delta_{z_{1}} g(z)$ and, as can be proved by induction on $l$ :

$$
\Delta_{z_{l} \ldots z_{1}}(g(z) h(z))=g\left(z_{1}\right) \Delta_{z_{l} \ldots z_{1}} h(z)+h(z) \Delta_{z_{l} \ldots z_{1}} g(z)
$$

$$
+\left.\sum_{n=1}^{l-1}\left(\Delta_{z_{n} \ldots z_{1}} g(z)\right)\right|_{z=z_{n+1}} \Delta_{z_{l} \ldots z_{n+1}} h(z) .
$$

Let $C_{1}$ denote the exterior of the interval $[1,+\infty)$ with respect to the finite complex plane and $C_{2}$ the exterior of the intervals $(-\infty,-1]$ and $[1,+\infty)$ with respect to the finite complex plane.

Then we can prove the following lemma due to A. Peyerimhoff [4] for which a different proof is given here:

Lemma 1. Let $f(z)=P_{k-1}(z)+z^{k} \int_{-1}^{1}(1 /(1-z t)) d g(t)$, where $P_{k-1}(z)$ is a real polynomial of degree $k-1$ at most $\left(P_{k-1}(z) \equiv 0\right.$ if $\left.k=0\right)$ and $g(t)$ is real and weakly monotone on $-1 \leqq t \leqq 1$ with $g(1) \neq g(-1)$.

Then $f(z)$ is holomorphic and has at most $k$ zeros in $C_{2}$. Similarly if $f(z)=P_{k-1}(z)$ $+z^{k} \int_{0}^{1}(1 /(1-z t)) d g(t)$, where $g(t)$ is real and weakly monotone on $0 \leqq t \leqq 1$, then $f(z)$ is holomorphic and has at most $k$ zeros in $C_{1}$.

Proof. We give a proof for the first part, the proof for the second part being similar.

If $k=0$, then $f(z)=\int_{-1}^{1}(1 /(1-z t)) d g(t)$.

If $z$ is real, $z=x, f(x)=\int_{-1}^{1}(1 /(1-x t)) d g(t) \neq 0$ for $-1<x<1$ since $g(1) \neq g(-1)$.

If $z=x+i y$ with $y \neq 0$, consider

$$
z f(z)=\int_{-1}^{1} \frac{z(1-\bar{z} t)}{|1-z t|^{2}} d g(t)=\int_{-1}^{1} \frac{x-|z|^{2} t}{|1-z t|^{2}} d g(t)+i y \int_{-1}^{1} \frac{1}{|1-z t|^{2}} d g(t) .
$$

Then

$$
\operatorname{Im}(z f(z))=y \int_{-1}^{1} \frac{1}{|1-z t|^{2}} d g(t) \neq 0
$$

since $y \neq 0$. Therefore $z f(z) \neq 0$ for $z \neq 0$ and $f(0)=g(1)-g(-1) \neq 0$. Therefore, if $k=0, f(z)$ has no zeros in $C_{2}$. 
Assume now $k \geqq 1$. Then $f(z)=P_{k-1}(z)+z^{k} \int_{-1}^{1}(1 /(1-z t)) d g(t)$ and we prove by induction on $k$ that for $z, z_{1}, z_{2}, \ldots, z_{k} \in C_{2}$ we have

$$
\Delta_{z_{k} \ldots z_{1}} f(z)=\int_{-1}^{1} \frac{1}{(1-z t)\left(1-z_{1} t\right) \cdots\left(1-z_{k} t\right)} d g(t) .
$$

This is trivial for $k=1$ and $\Delta_{z_{k} \ldots z_{1}}\left(P_{k-1}(z)\right)=0$ is trivial for any $k$.

Assume now that (2) holds for $k-1$ instead of $k$. Then

$$
\Delta_{z_{k-1} \ldots z_{1}}\left(\frac{z^{k-1}}{1-z t}\right)=\frac{1}{(1-z t)\left(1-z_{1} t\right) \cdots\left(1-z_{k-1} t\right)}
$$

Now apply (1) with $h(z)=z$ and $g(z)=z^{k-1} /(1-z t)$. Then

$$
\begin{aligned}
\Delta_{z_{k} \ldots z_{1}}\left(\frac{z^{k-1}}{1-z t} z\right) & =\left.\left(\Delta_{z_{k-1} \ldots z_{1}}\left(\frac{z^{k-1}}{1-z t}\right)\right)\right|_{z=z_{k}} \Delta_{z_{k}}(z)+z \Delta_{z_{k} \ldots z_{1}}\left(\frac{z^{k-1}}{1-z t}\right) \\
& =\frac{1}{\left(1-z_{k} t\right)\left(1-z_{1} t\right) \cdots\left(1-z_{k-1} t\right)}+\frac{z t}{(1-z t)\left(1-z_{1} t\right) \cdots\left(1-z_{k} t\right)} \\
& =\frac{1}{(1-z t)\left(1-z_{1} t\right) \cdots\left(1-z_{k} t\right)} \quad \text { which proves }(2) .
\end{aligned}
$$

Now we assume that $f(z)=P_{k-1}(z)+z^{k} \int_{-1}^{1}(1 /(1-z t)) d g(t)$ has at least $k$ zeros $z_{1}, \ldots, z_{k}$ in $C_{2}$. Then

(3) $\Delta_{z_{k} \ldots z_{1}} f(z)=\frac{f(z)}{\left(z-z_{1}\right) \cdots\left(z-z_{k}\right)}=\int_{-1}^{1} \frac{1}{(1-z t)\left(1-z_{1} t\right) \cdots\left(1-z_{k} t\right)} d g(t)$.

If $k$ is even we may assume that $z_{1}, \ldots, z_{k}$ consists of pairs of conjugate complex zeros and of real zeros of $f(z)$ in $C_{2}$. Then (3) gives:

$$
\frac{f(z)}{\left(z-z_{1}\right) \cdots\left(z-z_{k}\right)}=\int_{-1}^{1} \frac{1}{1-z t} d \gamma(t), \quad \text { where } \gamma(t)=\int_{-1}^{t} \frac{d g(\tau)}{\left(1-z_{1} \tau\right) \cdots\left(1-z_{k} \tau\right)}
$$

is a real monotone function for $-1 \leqq t \leqq 1$. According to what has been proved already $\int_{-1}^{1}(1 /(1-z t)) d \gamma(t)$ has no zeros in $C_{2}$ and so $f(z)$ does not have more zeros than $z_{1}, \ldots, z_{k}$.

If $k$ is odd and $f(z)$ has at least $k$ zeros in $C_{2}$, then $f(z)$ has at least 1 real zero in $C_{2}$. Otherwise $f(z)$ would have at least $k+1$ complex zeros $z_{1}, \ldots, z_{k}, z_{k+1}$ in $C_{2}$ which we can assume to consist of pairs of conjugate complex zeros. Especially we assume that $z_{k+1}=\bar{z}_{k}$. Then (3) gives .

$$
\frac{f(z)}{\left(z-z_{1}\right) \cdots\left(z-z_{k}\right)}=\int_{-1}^{1} \frac{1}{(1-z t)\left(1-z_{k} t\right)} d \gamma(t)
$$

where

$$
\gamma(t)=\int_{-1}^{t} \frac{d g(\tau)}{\left(1-z_{1} \tau\right) \cdots\left(1-z_{k-1} \tau\right)}
$$

is a real monotone function for $-1 \leqq t \leqq 1$. 
In (4) the left side is holomorphic in $C_{2}$ and vanishes at $z=z_{k+1}=\bar{z}_{k}$ whereas the right side is $\int_{-1}^{1}\left(1 /\left|1-z_{k} t\right|^{2}\right) d \gamma(t) \neq 0$. Therefore, if $f(z)$ has at least $k$ zeros in $C_{2}$, where $k$ is odd, $f(z)$ has at least one real zero in $C_{2}$. Now we apply the same argument as in the case where $k$ was even to the $k$ zeros $z_{1}, \ldots, z_{k}$ in $C_{2}$, where we now can assume that $z_{1}, \ldots, z_{k}$ ( $k$ odd) consists of pairs of conjugate complex and of real zeros (at least one) of $f(z)$ in $C_{2}$. This again shows that $f(z)$ cannot have more than $k$ zeros in $C_{2}$.

LEMMA 2. If $P_{l}(z)$ is a real polynomial of degree $l$ and $g(t)$ is real and weakly monotone for $-1 \leqq t \leqq 1$, then for $z \in C_{2}$

$$
P_{l}(z) \int_{-1}^{1} \frac{1}{1-z t} d g(t)=P_{l-1}(z)+z^{l} \int_{-1}^{1} \frac{t^{l} P_{l}(1 / t)}{1-z t} d g(t)
$$

where $P_{l-1}(z)$ is a real polynomial of degree $l-1$ at most.

$A$ similar formula holds if the integrals from -1 to 1 are replaced by integrals from 0 to 1 , and if $C_{2}$ is replaced by $C_{1}$.

Proof $\left({ }^{2}\right) . h(z)=P_{l}(z)-z^{l} t^{l} P_{l}(1 / t)$ is a polynomial in $z$ of degree $l$ at most which vanishes at $z=1 / t$. Therefore $h(z) /(1-z t)$ is a real polynomial in $z$ of degree $l-1$ at most.

The same is true of $\int_{-1}^{1}(h(z) /(1-z t)) d g(t)$.

LEMMA 3. If $l<k$ and $g(t)$ is real and weakly monotone for $-1 \leqq t \leqq 1$ then

$$
z^{l} \int_{-1}^{1} \frac{1}{1-z t} d g(t)=P_{k-1}(z)+z^{k} \int_{-1}^{1} \frac{t^{k-l}}{1-z t} d g(t) \text { for } z \in C_{2}
$$

A similar formula holds for $z \in C_{1}$ if the integrals from -1 to 1 are replaced by integrals from 0 to 1.

$\operatorname{Proof}\left({ }^{2}\right) . h(z)=z^{l}-z^{k} t^{k-l}$ is a real polynomial in $z$ of degree $k$ at most which vanishes at $z=1 / t$.

Therefore $h(z) /(1-z t)$ is a real polynomial in $z$ of degree $k-1$ at most. The same is true of $\int_{-1}^{1}(h(z) /(1-z t)) d g(t)$.

2. We assume that $f(z)$ is an entire function of finite order $\rho$ which is real for real $z$ and which has finitely many complex zeros and infinitely many real zeros.

If $r_{n}(n=1,2, \ldots)$ are the absolute values in increasing order of the zeros $z_{n} \neq 0$ of $f(z)$ then there exists a smallest nonnegative integer $p$ (the genus of the sequence $r_{n}$ ) such that $\sum_{n=1}^{\infty} 1 / r_{n}^{p}=\infty$ and $\sum_{n=1}^{\infty} 1 / r_{n}^{p+1}<\infty$. Then always $p \leqq \rho$ and $p=[\rho]$ if $\rho$ is not an integer [6]. The convergence exponent $\sigma$ of $r_{n}$ is the greatest lower bound of positive numbers $\alpha$ such that $\sum_{n=1}^{\infty} 1 / r_{n}^{\alpha}<\infty$. Then always $p \leqq \sigma \leqq p+1$.

$\left.{ }^{2}\right)$ I am indebted to the referee for simplifying the proofs of Lemmas 2 and 3. 
From Hadamard's factorization theorem [6] follows that $f(z)$ can be written as

$$
f(z)=z^{k} e^{Q(z)} \prod_{n=1}^{\infty}\left(1-\frac{z}{z_{n}}\right) \exp \left[z / z_{n}+\left(z / z_{n}\right)^{2} / 2+\cdots+\left(z / z_{n}\right)^{p} / p\right]
$$

where $Q(z)$ is a real polynomial of degree $q$ with $q \leqq[\rho]$ and

$$
\prod_{n=1}^{\infty}\left(1-\frac{z}{z_{n}}\right) \exp \left[z / z_{n}+\cdots+\left(z / z_{n}\right)^{p} / p\right]
$$

is the canonical product of genus $p$ associated with the zeros $z_{n}$ which, as an entire function is of order $\sigma$ [6]. Then always $\rho=\max (q, \sigma)[6]$.

Now we consider the entire function (of order $\leqq \rho$ ).

$g(z)=A(z) f(z)+B(z) f^{\prime}(z)$ where $A(z)$ and $B(z)$ are real polynomials of degrees $a$ and $b$ respectively, where we agree that always $b \geqq 0$ and $A(z) \equiv 0$ if $a=-1$.

With this notation we have the following

THEOREM 1.

Case I. Let $f(z)$ have infinitely many positive, finitely many negative and finitely many complex zeros $(f(z)$ may have a zero at $z=0)$.

Let $a_{1}$ denote a real zero of $f(z)$ with multiplicity $\alpha_{1}$ such that all real zeros of $B(z)$ are less than $a_{1}$. All real zeros of $f(z)$ which are bigger than $a_{1}$ are denoted by $a_{n}$ in increasing order with multiplicities $\alpha_{n}(n=2,3, \ldots)$. If $B(z)$ does not have any real zeros, $a_{1}$ can be any real zero of $f(z)$.

Then at each point $a_{n}(n=1,2, \ldots), g(z)$ has a zero of multiplicity $\alpha_{n}-1$ exactly and between each pair $a_{n}, a_{n+1}(n=1,2, \ldots) g(z)$ has an odd number of zeros.

Let $k$ be the number of zeros of $f(z)$ which are different from $a_{n}(n=1,2, \ldots)$. Then the number of zeros of $g(z)$ besides the trivial zeros at $a_{n}$ and besides one zero between each pair $a_{n}, a_{n+1}(n=1,2, \ldots)$ is

1. exactly $b+p+k$ if $p \leqq \rho<p+1$ and $a \leqq b+p-1$ or if $\rho=\sigma=p+1, q \leqq p$ and $a \leqq b+p-1$,

2. at most $b+\rho+k \quad$ if $\rho$ does not satisfy 1 and if $a \leqq b+\rho-1$,

3. at most $a+k+1 \quad$ if a does not satisfy 1 or 2 .

(Always $p<\rho<p+1$ is true if $\rho$ is not an integer.)

Case II. Let $f(z)$ have infinitely many positive, infinitely many negative and finitely many complex zeros $(f(z)$ may have a zero at $z=0)$.

Let $a_{1}$ denote a real zero of $f(z)$ with multiplicity $\alpha_{1}$ such that all real zeros of $B(z)$ are less than $a_{1}$. All real zeros of $f(z)$ which are bigger than $a_{1}$ are denoted by $a_{n}$ in increasing order and with multiplicities $\alpha_{n}(n=2,3, \ldots)$. Similarly let $-b_{1}$ denote a real zero of $f(z)$ with multiplicity $\beta_{1}$ such that all real zeros of $B(z)$ are bigger than $-b_{1}$. All real zeros of $f(z)$ which are smaller than $-b_{1}$ are denoted by $-b_{n}$ in decreasing order with multiplicities $\beta_{n}(n=2,3, \ldots)$. If $B(z)$ does not have any real zeros, $-b_{1}$ and $a_{1}$ can be arbitrary real zeros of $f(z)$ with $-b_{1}<a_{1}$.

Then at each point $a_{n}$ and $-b_{n}(n=1,2, \ldots) g(z)$ has a zero of exact multiplicity $\alpha_{n}-1$ and $\beta_{n}-1$ respectively and an odd number of zeros between each pair $a_{n}, a_{n+1}$ and $-b_{n+1},-b_{n}(n=1,2, \ldots)$. 
Let $k$ be the number of zeros of $f(z)$ which are different from $a_{n}$ and $-b_{n}(n=1$, $2, \ldots)$.

Then the number of zeros of $g(z)$ besides the trivial zeros at $a_{n}$ and $-b_{n}$ and besides one zero between each pair $a_{n}, a_{n+1}$ and $-b_{n+1},-b_{n}(n=1,2, \ldots)$ is
1. exactly $p+b+k$
if $p$ is odd, $p \leqq \rho<p+1$ and $a \leqq b+p-1$, or
if $\rho=\sigma=p+1, p \geqq q$ and $a \leqq b+p-1$,
2. exactly $p+b+k+1$
if $p$ is even, $p \leqq \rho \leqq p+1, a \leqq b+p$,
3. at most $b+k+\rho$
if $\rho$ is odd and does not satisfy 1 or $2, a \leqq b+\rho-1$,
4. at most $b+k+\rho+1$
if $\rho$ is even and does not satisfy 1 or $2, a \leqq b+\rho$,
5. at most $a+k+1$
if $a-b$ is even and $a$ does not satisfy $1-4$,
6. at most $a+k+2$
if $a-b$ is odd and a does not satisfy 1-4.
(Always $p<\rho<p+1$ is true if $\rho$ is not an integer.)

REMARKS. A similar result as in Case I holds for functions $f(z)$ which have infinitely many negative and finitely many positive zeros. One only has to replace $g(z)$ by $g(-z)$.

The results in Cases I and II remain correct if $B(z)$ does have real zeros of even order between zeros $a_{n}, a_{n+1}$ and $-b_{n+1},-b_{n}$ as long as they are different from all $a_{n}$ and $-b_{n}$.

Proof. In the following we assume that $a_{1}=1$ and $-b_{1}=-1$. This can be assumed without loss of generality, since we can replace the variable $z$ by $\alpha z+\beta$ where $\alpha, \beta$ are suitable real constants.

From the assumptions made about $f(z)$ it follows that according to Hadamard's factorization theorem in Case I $f(z)$ can be written as $f(z)=K(z) e^{Q(z)} \Pi(z)$, where

$$
\Pi(z)=\prod_{n=1}^{\infty}\left(1-\frac{z}{a_{n}}\right)^{\alpha_{n}} \exp \left[\alpha_{n}\left(\frac{z}{a_{n}}+\cdots+\frac{1}{p}\left(\frac{z}{a_{n}}\right)^{p}\right)\right]
$$

and in Case II as $f(z)=K(z) e^{Q(z)} \Pi_{1}(z) \Pi_{2}(z)$, where

and

$$
\Pi_{1}(z)=\prod_{n=1}^{\infty}\left(1-\frac{z}{a_{n}}\right)^{\alpha_{n}} \exp \left[\alpha_{n}\left(\frac{z}{a_{n}}+\cdots+\frac{1}{p}\left(\frac{z}{a_{n}}\right)^{p}\right)\right]
$$

$$
\Pi_{2}(z)=\prod_{n=1}^{\infty}\left(1+\frac{z}{b_{n}}\right)^{\beta_{n}} \exp \left[\beta_{n}\left(-\frac{z}{b_{n}}+\cdots+\frac{(-1)^{p}}{p}\left(\frac{z}{b_{n}}\right)^{p}\right)\right] .
$$

In Case I $K(z)$ is a real polynomial of degree $k$, whose zeros coincide with all zeros of $f(z)$ which are different from $a_{n}(n=1,2, \ldots) . Q(z)$ is a real polynomial of degree $q$ with $q \leqq[\rho] . p$ is the genus of the sequence $a_{n}$.

In Case II $K(z)$ is a real polynomial of degree $k$ whose zeros coincide with all zeros of $f(z)$ which are different from $a_{n}$ and $-b_{n}(n=1,2, \ldots) . Q(z)$ is as in Case I and $p$ is defined by

$$
\sum_{n=1}^{\infty} \frac{\alpha_{n}}{a_{n}^{p}}+\frac{\beta_{n}}{b_{n}^{p}}=\infty \quad \text { and } \quad \sum_{n=1}^{\infty} \frac{\alpha_{n}}{a_{n}^{p+1}}+\frac{\beta_{n}}{b_{n}^{p+1}}<\infty .
$$


Substituting the expressions for $f(z)$ in $g(z)=A(z) f(z)+B(z) f^{\prime}(z)$ we obtain in Case I

$$
g=e^{Q} \Pi\left(A K+B K^{\prime}+B K Q^{\prime}+B K\left(\Pi^{\prime} / \Pi\right)\right)
$$

and in Case II

$$
g=e^{Q} \Pi_{1} \Pi_{2}\left[A K+B K^{\prime}+B K Q^{\prime}+B K\left(\left(\Pi_{1}^{\prime} / \Pi_{1}\right)+\left(\Pi_{2}^{\prime} / \Pi_{2}\right)\right)\right] .
$$

Here

$$
\frac{\Pi^{\prime}}{\Pi}=\sum_{n=1}^{\infty}\left(\frac{\alpha_{n}}{z-a_{n}}+\alpha_{n}\left(\frac{1}{a_{n}}+\cdots+\frac{z^{p-1}}{a_{n}}\right)\right) \text { or } \frac{\Pi^{\prime}}{\Pi}=z^{p} \sum_{n=1}^{\infty} \frac{\alpha_{n}}{a_{n}^{p}\left(z-a_{n}\right)} .
$$

Therefore in Case I

$$
g=e^{Q} \Pi\left(A K+B K^{\prime}+B K Q^{\prime}+B K z^{p}\left(\sum_{n=1}^{\infty} \frac{\alpha_{n}}{a_{n}^{p}\left(z-a_{n}\right)}\right)\right)
$$

and similarly in Case II

(3) $g=e^{Q} \Pi_{1} \Pi_{2}\left(A K+B K^{\prime}+B K Q^{\prime}+B K z^{p}\left(\sum_{n=1}^{\infty} \frac{\alpha_{n}}{a_{n}^{p}\left(z-a_{n}\right)}+(-1)^{p} \sum_{n=1}^{\infty} \frac{\beta_{n}}{b_{n}^{p}\left(z+b_{n}\right)}\right)\right)$.

Since in Case I $B(z)$ and $K(z)$ do not have zeros for $z \geqq a_{1}$ it follows that $g(z)$ has an odd number of zeros between each pair $a_{n}, a_{n+1}(n=1,2, \ldots)$. Similarly in Case II $B(z)$ and $K(z)$ do not have zeros for $z \geqq a_{1}$ and $z \leqq-b_{1}$, so that $g(z)$ has an odd number of zeros between each pair $a_{n}, a_{n+1}$ and $-b_{n+1}, \cdots-b_{n}(n=1,2, \ldots)$.

From now on we assume without loss of generality that in Case I $B(z) K(z)>0$ for $z \geqq a_{1}$ (otherwise replace $g(z)$ by $-g(z)$ ) and in Case II that (see the definition of $p$ in Case II) $\sum_{n=1}^{\infty} \alpha_{n} / a_{n}^{p}=\infty$ and $\sum_{n=1}^{\infty} \alpha_{n} / a_{n}^{p+1}<\infty$, and that at the same time $B(z) K(z)>0$ for $z \geqq a_{1}$ (otherwise replace $g(z)$ by $g(-z)$ and/or $g(z)$ by $-g(z)$ ).

Now in Case I

$$
\sum_{n=1}^{\infty} \frac{\alpha_{n}}{a_{n}^{p}\left(z-a_{n}\right)}=-\sum_{n=1}^{\infty} \frac{1}{1-z\left(1 / a_{n}\right)}\left(\frac{\alpha_{n}}{a_{n}^{p+1}}\right)=-\int_{0}^{1} \frac{1}{1-z t} d \gamma(t)
$$

with $\gamma(t)=\sum_{1 / a_{n} \leqq t} \alpha_{n} / a_{n}^{p+1}$ for $0 \leqq t \leqq 1, \gamma(t)$ being nondecreasing for $0 \leqq t \leqq 1$. This gives

$$
g=e^{Q} \Pi\left(A K+B K^{\prime}+B K Q^{\prime}-B K z^{p} \int_{0}^{1} \frac{1}{1-z t} d \gamma(t)\right) .
$$

We now use Lemma 2 to obtain

$$
B(z) K(z) \int_{0}^{1} \frac{1}{1-z t} d \gamma(t)=P_{b+k-1}(z)+z^{b+k} \int_{0}^{1} \frac{t^{b+k} B(1 / t) K(1 / t)}{1-z t} d \gamma(t)
$$

where $P_{b+k-1}(z)$ is a real polynomial of degree $b+k-1$ at most. With $\eta(t)=$ $\int_{0}^{t} \tau^{b+k} B(1 / \tau) K(1 / \tau) d \gamma(\tau), \eta(t)$ being nondecreasing on $0 \leqq t \leqq 1$, we finally obtain in Case I

$$
g=e^{Q} \Pi\left(A K+B K^{\prime}+B K Q^{\prime}-z^{p} P_{b+k-1}(z)-z^{p+b+k} \int_{0}^{1} \frac{1}{1-z t} d \eta(t)\right) .
$$


In Case II we put

$$
\begin{gathered}
\sum_{n=1}^{\infty} \frac{\alpha_{n}}{a_{n}^{p}\left(z-a_{n}\right)}=-\int_{0}^{1} \frac{1}{1-z t} d \gamma_{1}(t) \quad \text { with } \gamma_{1}(t)=\sum_{1 / a_{n} \leqq t} \frac{\alpha_{n}}{a_{n}^{p+1}} \text { for } 0 \leqq t \leqq 1, \\
\sum_{n=1}^{\infty} \frac{\beta_{n}}{b_{n}^{p}\left(z+b_{n}\right)}=\int_{0}^{1} \frac{1}{1+z t} d \gamma_{2}(t) \quad \text { with } \gamma_{2}(t)=\sum_{1 / b_{n} \leqq t} \frac{\beta_{n}}{b_{n}^{p+1}} \text { for } 0 \leqq t \leqq 1,
\end{gathered}
$$

$\gamma_{1}(t), \gamma_{2}(t)$ being nondecreasing for $0 \leqq t \leqq 1$.

Now

$$
\int_{0}^{1} \frac{1}{1+z t} d \gamma_{2}(t)=\int_{-1}^{0} \frac{1}{1-z t} d\left(-\gamma_{2}(-t)\right)
$$

and from (3) follows

$$
\begin{aligned}
g=e^{Q} \Pi_{1} \Pi_{2}\left(A K+B K^{\prime}\right. & +B K Q^{\prime}-B K z^{p} \\
& \left.\times\left(\int_{0}^{1} \frac{1}{1-z t} d \gamma_{1}(t)+\int_{-1}^{0} \frac{1}{1-z t} d(-1)^{p} \gamma_{2}(-t)\right)\right) .
\end{aligned}
$$

Here $(-1)^{p} \gamma_{2}(-t)$ is increasing for $-1 \leqq t \leqq 0$ if $p$ is odd and decreasing if $p$ is even. Define

$$
\begin{aligned}
\gamma(t) & =-\gamma_{2}(-t) & & \text { for }-1 \leqq t \leqq 0 \\
& =\gamma_{1}(t) & \text { for } 0 \leqq t \leqq 1 & \text { if } p \text { is odd }
\end{aligned}
$$

and

$$
\begin{array}{rlrl}
\gamma(t) & =\gamma_{2}(-t) & & \text { for }-1 \leqq t \leqq 0 \\
& =\gamma_{1}(t) & \text { for } 0 \leqq t \leqq 1
\end{array} \quad \text { if } p \text { is even }
$$

Then (5) gives

$$
g=e^{Q} \Pi_{1} \Pi_{2}\left(A K+B K^{\prime}+B K Q^{\prime}-B K z^{p} \int_{-1}^{1} \frac{1}{1-z t} d \gamma(t)\right)
$$

Using Lemma 2 again we have

$$
B(z) K(z) \int_{-1}^{1} \frac{1}{1-z t} d \gamma(t)=P_{b+k-1}(z)+z^{b+k} \int_{-1}^{1} \frac{t^{b+k} B(1 / t) K(1 / t)}{1-z t} d \gamma(t)
$$

where $P_{b+k-1}(z)$ is a real polynomial of degree $b+k-1$ at most.

If $p$ is even we use Lemma 3 in addition to obtain

$$
\int_{-1}^{1} \frac{t^{b+k} B(1 / t) K(1 / t)}{1-z t} d \gamma(t)=c+z \int_{-1}^{1} \frac{t^{b+k+1} B(1 / t) K(1 / t)}{1-z t} d \gamma(t)
$$

where $c$ is a real constant.

Finally we put

$$
\begin{array}{ll}
\eta(t)=\int_{-1}^{t} \tau^{b+k} B(1 / \tau) K(1 / \tau) d \gamma(\tau) & \text { for }-1 \leqq t \leqq 1 \text { if } p \text { is odd } \\
\eta(t)=\int_{-1}^{t} \tau^{b+k+1} B(1 / \tau) K(1 / \tau) d \gamma(\tau) & \text { for }-1 \leqq t \leqq 1 \text { if } p \text { is even. }
\end{array}
$$


Then we obtain from (6)

$$
g=e^{Q} \Pi_{1} \Pi_{2}\left(A K+B K^{\prime}+B K Q^{\prime}-z^{p} P_{b+k-1}-z^{p+b+k} \int_{-1}^{1} \frac{1}{1-z t} d \eta(t)\right)
$$

if $p$ is odd,

$$
\begin{aligned}
& g=e^{Q} \Pi_{1} \Pi_{2}\left(A K+B K^{\prime}+B K Q^{\prime}-z^{p} P_{b+k-1}-c z^{p+b+k}\right. \\
& \left.-z^{p+b+k+1} \int_{-1}^{1} \frac{1}{1-z t} d \eta(t)\right) \text { if } p \text { is even. }
\end{aligned}
$$

From the assumptions made about $B(z) K(z)$ without loss of generality it follows that $\eta(t)$ is an increasing function for $-1 \leqq t \leqq 1$.

In Case I we obtain

$$
g=e^{Q} \Pi\left(P(z)-z^{l} \int_{0}^{1} \frac{1}{1-z t} d \eta(t)\right)
$$

and in Case II

$$
g=e^{Q} \Pi_{1} \Pi_{2}\left(P(z)-z^{l} \int_{-1}^{1} \frac{1}{1-z t} d \eta(t)\right) \text { if } p \text { is odd }
$$

and

$$
g=e^{Q} \Pi_{1} \Pi_{2}\left(P(z)-z^{l+1} \int_{-1}^{1} \frac{1}{1-z t} d \eta(t)\right) \text { if } p \text { is even. }
$$

Here $l=p+b+k$ and in all three cases $P(z)$ is a real polynomial.

To these three expressions we apply Lemma 3, if necessary, and then Lemma 1 in order to obtain that in $C_{1}$ and in $C_{2}$ respectively $g(z)$ has at most as many zeros as stated in Theorem 1.

If now the same proof is carried out with $a_{2}$ instead of $a_{1}$, so that $k$ has to be replaced by $k+\alpha_{1}$, the upper bound of zeros of $g(z)$ in $C_{1}^{*}$ and $C_{2}^{*}$ respectively, which is given by Lemma 1 , is increased by $\alpha_{1}$. But in the statement of Theorem 1 these additional $\alpha_{1}$ zeros are already counted with the trivial $\left(\alpha_{1}-1\right)$-fold zero of $g(z)$ at $a_{1}$ and with one zero of $g(z)$ between $a_{1}$ and $a_{2}$. Here the ${ }^{*}$ in $C_{1}^{*}$ and $C_{2}^{*}$ refers to the fact that now we assume without loss of generality that $a_{2}=1$.

This argument can be repeated with all $a_{n}(n=3,4, \ldots)$ and all $-b_{n}(n=2,3, \ldots)$, which shows that besides the trivial zeros $g(z)$ has at most as many zeros as stated in Theorem 1.

It remains to show that in some special cases listed in Theorem 1 the upper bound for the number of zeros of $g(z)$ actually is the exact number of zeros.

In Case I with $l=p+b+k$ we obtained in (4) that

$$
g(z)=e^{Q(z)} \Pi(z)\left(P_{l-1}(z)-z^{l} \int_{0}^{1} \frac{1}{1-z t} d \eta(t)\right)
$$

if $p \leqq \rho<p+1$ and $a \leqq b+p-1$, or $\sigma=\rho=p+1, q \leqq p$, and $a \leqq b+p-1$. Here $P_{l-1}(z)$ is a real polynomial of degree $l-1$ at most. 
We now consider

$$
h(z, \tau)=\tau P_{l-1}(z)-z^{l} \int_{0}^{1} \frac{1}{1-z t} d \eta(t) \text { for } 0 \leqq \tau \leqq 1 .
$$

Then

$$
h(z, \tau)=\tau P_{l-1}(z)+z^{p} P_{b+k-1}(z)+B(z) K(z) z^{p} \sum_{n=1}^{\infty} \frac{\alpha_{n}}{a_{n}^{p}\left(z-a_{n}\right)},
$$

which shows that for each $\tau, 0 \leqq \tau \leqq 1, h(z, \tau)$ has a simple pole at $a_{n}$ and an odd number of zeros between each pair $a_{n}, a_{n+1}(n=1,2, \ldots)$.

We now want to determine a circle $S$ around the origin such that $h(z, \tau) \neq 0$ for $z \in S$ and $0 \leqq \tau \leqq 1$. With $z=r e^{i \theta}$ we have for $r \geqq 1$ and all $0 \leqq \tau \leqq 1$

$$
\begin{aligned}
\left|\frac{h(z, \tau)}{z^{l-1}}\right| & \geqq\left|\operatorname{Im} \frac{h(z, \tau)}{z^{l-1}}\right| \geqq\left|\operatorname{Im} \int_{0}^{1} \frac{z(1-\bar{z} t)}{|1-z t|^{2}} d \eta(t)\right|-\left|\operatorname{Im} \tau \frac{P_{l-1}(z)}{z^{l-1}}\right| \\
& \geqq r|\sin \theta|\left(\int_{0}^{1} \frac{d \eta(t)}{|1-z t|^{2}}-\frac{c_{1}}{r^{2}}\right) \geqq r|\sin \theta|\left(\int_{0}^{1} \frac{t^{b+k} B(1 / t) K(1 / t)}{(1+r t)^{2}} d \gamma(t)-\frac{c_{1}}{r^{2}}\right) \\
& \geqq r|\sin \theta|\left(c_{2} \int_{0}^{1} \frac{d \gamma(t)}{(1+r t)^{2}}-\frac{c_{1}}{r^{2}}\right) \text { with } c_{1}, c_{2}>0,
\end{aligned}
$$

using the fact that $t^{b+k} B(1 / t) K(1 / t) \geqq c_{2}>0$ for $0 \leqq t \leqq 1$.

Here

$$
\int_{0}^{1} \frac{d \gamma(t)}{(1+r t)^{2}} \geqq \int_{1 / r}^{1} \frac{d \gamma(t)}{(1+r t)^{2}} \geqq \int_{1 / r}^{1} \frac{d \gamma(t)}{(2 r t)^{2}} \geqq \frac{1}{4 r^{2}} \int_{1 / r}^{1} \frac{d \gamma(t)}{t} .
$$

Therefore

$$
|h(z, \tau)| \geqq|z|^{l-2}|\sin \theta|\left(\frac{c_{2}}{4} \int_{1 / r}^{1} \frac{d \gamma(t)}{t}-c_{1}\right) .
$$

From the definition of $\gamma(t)$ follows

$$
\int_{1 / r}^{1} \frac{d \gamma(t)}{t}=\sum_{r \geqq a_{n}} \frac{\alpha_{n}}{a_{n}^{p}}
$$

and therefore $\lim _{r \rightarrow \infty} \int_{1 / r}^{1} d \gamma(t) / t=\infty$. This shows that for $0 \leqq \tau \leqq 1$

$$
|h(z, \tau)|>0 \text { if } r \text { is sufficiently large and } \sin \theta \neq 0 \text {. }
$$

If $z=-r$ then for all $r \geqq 1$ and $0 \leqq \tau \leqq 1$

$$
|h(-r, \tau)| \geqq r^{l-1}\left(r \int_{0}^{1} \frac{1}{1+r t} d \eta(t)-c_{3}\right) \geqq r^{l-1}\left(c_{2} \int_{0}^{1} \frac{r}{1+r t} d \gamma(t)-c_{3}\right)
$$

which is $>0$ if $r$ is sufficiently large according to $(8)\left({ }^{3}\right)$. The constants $c_{2}, c_{3}$ are $>0$.

$\left({ }^{3}\right)$ Since $r \int_{0}^{1}(d \gamma(t) /(1+r t)) \geqq \frac{1}{2} \int_{1 / r}^{1}(d \gamma(t) / t)$. 
If $z=r$, we choose $r_{1}$ large enough and close to, but $<a_{m}$ for some $m \geqq 1$, so that (9) holds for $0<\theta<2 \pi$ and such that

$$
|h(r, \tau)| \geqq\left|r^{l} \int_{0}^{1} \frac{d \eta(t)}{1-r t}\right|-\left|P_{l-1}(r)\right|>0 \text { for } r_{1} \leqq r<a_{m} \text { and } 0 \leqq \tau \leqq 1 .
$$

This is possible since $h(z, \tau)$ has a simple pole at each point $a_{n}(n=1,2, \ldots)$. Let now $S$ be the circle around the origin with this radius $r_{1}$. Then $|h(z, \tau)|>0$ for all $z \in S$ and $0 \leqq \tau \leqq 1$ so that $|h(z, \tau)| \geqq c_{4}>0$ for these $z$ and $\tau$, since $|h(z, \tau)|$ is continuous as a function of $(z, \tau) \in S \times[0,1]$.

Therefore $(1 / 2 \pi i) \int_{S}\left(h^{\prime}(z, \tau) / h(z, \tau)\right) d z$ is continuous as a function of $\tau$ for $0 \leqq \tau \leqq 1$ and therefore a constant. Since $h(z, 0)$ has an $l$-fold 0 at $z=0$ and at least one zero between each pair of poles $a_{n}, a_{n+1}$ and since $h(z, \tau)$ has at least one zero between each pair $a_{n}, a_{n+1}(n=1, \ldots, m-1)$ for all $0 \leqq \tau \leqq 1$, we have

$$
\frac{1}{2 \pi i} \int_{S} \frac{h^{\prime}(z, \tau)}{h(z, \tau)} d z \geqq l
$$

Together with what has been proved earlier we obtain that $h(z, 1)$ or $g(z)=$ $e^{Q(z)} \Pi(z) h(z, 1)$ has exactly $l$ zeros besides one zero between each pair $a_{n}, a_{n+1}$ $(n=1,2, \ldots)$ and besides trivial zeros at $a_{n}$ of multiplicity $\alpha_{n}-1$.

In Case II with $l=p+b+k$ we have obtained in (7)

$$
\begin{aligned}
& g(z)=e^{Q} \Pi_{1} \Pi_{2}\left(P_{l-1}(z)-z^{l} \int_{-1}^{1} \frac{1}{1-z t} d \eta(t)\right) \text { if } p \text { is odd, } \\
& g(z)=e^{Q} \Pi_{1} \Pi_{2}\left(P_{l}(z)-z^{l+1} \int_{-1}^{1} \frac{1}{1-z t} d \eta(t)\right) \text { if } p \text { is even }
\end{aligned}
$$

and if the additional conditions of 1 or 2 in Case II of Theorem 1 are satisfied. Here $P_{l-1}(z)$ and $P_{l}(z)$ are real polynomials of degrees $l-1$ and $l$ at most.

We now consider, similarly as in Case I for $0 \leqq \tau \leqq 1$

$$
\begin{aligned}
h(z, \tau)= & \tau P_{l-1}(z)-z^{l} \int_{-1}^{1} \frac{1}{1-z t} d \eta(t) \\
= & \tau P_{l-1}(z)+z^{p} P_{b+k-1}+z^{p} B(z) K(z) \\
& \times\left(\sum_{n=1}^{\infty} \frac{\alpha_{n}}{a_{n}^{p}\left(z-a_{n}\right)}+(-1)^{p} \sum_{n=1}^{\infty} \frac{\beta_{n}}{b_{n}^{p}\left(z+b_{n}\right)}\right)
\end{aligned}
$$

if $p$ is odd and

$$
\begin{aligned}
h(z, \tau)= & \tau P_{l}(z)-z^{l+1} \int_{-1}^{1} \frac{1}{1-z t} d \eta(t) \\
= & \tau P_{l}(z)+c z^{l}+z^{p} P_{b+k-1}+z^{p} B(z) K(z) \\
& \times\left(\sum_{n=1}^{\infty} \frac{\alpha_{n}}{a_{n}^{p}\left(z-a_{n}\right)}+(-1)^{p} \sum_{n=1}^{\infty} \frac{\beta_{n}}{b_{n}^{p}\left(z+b_{n}\right)}\right)
\end{aligned}
$$

if $p$ is even. 
In both cases $h(z, \tau)$ has for each $\tau, 0 \leqq \tau \leqq 1$, a simple pole at $a_{n}$ and $-b_{n}$ $(n=1,2, \ldots)$ and an odd number of zeros between each pair $a_{n}, a_{n+1}$ and $-b_{n+1}$, $-b_{n}$. Similarly as in Case I we now want to determine a curve $S$ around the origin such that $h(z, \tau) \neq 0$ for $z \in S$ and $0 \leqq \tau \leqq 1$.

With $z=r e^{i \theta}$ we have

(12) $\left|\frac{h(z, \tau)}{z^{l-1}}\right| \geqq\left|\operatorname{Im} \frac{h(z, \tau)}{z^{l-1}}\right| \geqq r|\sin \theta|\left(\int_{-1}^{1} \frac{1}{|1-z t|^{2}} d \eta(t)-\frac{c_{1}}{r^{2}}\right)$ if $p$ is odd,

(13) $\left|\frac{h(z, \tau)}{z^{l}}\right| \geqq\left|\operatorname{Im} \frac{h(z, \tau)}{z^{l}}\right| \geqq r|\sin \theta|\left(\int_{-1}^{1} \frac{1}{|1-z t|^{2}} d \eta(t)-\frac{c_{2}}{r^{2}}\right)$ if $p$ is even.

This holds for all $r \geqq 1$ and all $0 \leqq \tau \leqq 1$ with constants $c_{1}$ and $c_{2}$ which are $>0$.

Now we use the fact that (w.l.o.g.) we have assumed $\sum_{n=1}^{\infty} \alpha_{n} / a_{n}^{p}=\infty$ and $\sum_{n=1}^{\infty} \alpha_{n} / a_{n}^{p+1}<\infty$ and that $t^{b+k} B(1 / t) K(1 / t) \geqq c_{3}>0$ for $-1 \leqq t \leqq 1$. Then in both expressions (12), (13) we have if $p$ is odd

$$
\begin{aligned}
\int_{-1}^{1} \frac{1}{|1-z t|^{2}} d \eta(t) & =\int_{-1}^{1} \frac{t^{b+k} B(1 / t) K(1 / t)}{|1-z t|^{2}} d \gamma(t) \geqq c_{3} \int_{0}^{1} \frac{d \gamma_{1}(t)}{|1-z t|^{2}} \geqq c_{3} \int_{0}^{1} \frac{d \gamma_{1}(t)}{(1+r t)^{2}} \\
& \geqq c_{3} \int_{1 / r}^{1} \frac{d \gamma_{1}(t)}{(2 r t)^{2}} \geqq \frac{c_{3}}{4 r^{2}} \int_{1 / r}^{1} \frac{d \gamma_{1}(t)}{t} .
\end{aligned}
$$

Similarly if $p$ is even

$$
\int_{-1}^{1} \frac{1}{|1-z t|^{2}} d \eta(t)=\int_{-1}^{1} \frac{t^{b+k+1} B(1 / t) K(1 / t)}{|1-z t|^{2}} d \gamma(t) \geqq c_{3} \int_{0}^{1} \frac{t d \gamma_{1}(t)}{|1-z t|^{2}}
$$

and now this is

$$
\geqq \frac{c_{3}}{4 r^{2}} \int_{1 / r}^{1} \frac{t d \gamma_{1}(t)}{t^{2}}=\frac{c_{3}}{4 r^{2}} \int_{1 / r}^{1} \frac{d \gamma_{1}(t)}{t} .
$$

Consequently we obtain for all $0 \leqq \tau \leqq 1$ and $r \geqq 1$ from (12), (13)

$$
\begin{aligned}
& |h(z, \tau)| \geqq r^{l-2}|\sin \theta|\left(\frac{c_{3}}{4} \int_{1 / r}^{1} \frac{d \gamma_{1}(t)}{t}-c_{1}\right) \quad \text { if } p \text { is odd, } \\
& |h(z, \tau)| \geqq r^{l-1}|\sin \theta|\left(\frac{c_{3}}{4} \int_{1 / r}^{1} \frac{d \gamma_{1}(t)}{t}-c_{2}\right) \quad \text { if } p \text { is even. }
\end{aligned}
$$

Similarly as in Case I $\int_{1 / r}^{1} d \gamma_{1}(t) / t=\sum_{a_{n} \leqq r} \alpha_{n} / a_{n}^{p}$ and therefore $\lim _{r \rightarrow \infty} \int_{1 / r}^{1} d \gamma_{1}(t) / t$ $=\infty$ so that

$$
|h(z, \tau)|>0 \text { for all } 0 \leqq \tau \leqq 1 \text { if } r \text { is sufficiently large and } \sin \theta \neq 0 .
$$

We then choose $r_{1}$ large enough and close to, but $<a_{m_{1}}$ for some $m_{1} \geqq 1$, such that for all $0 \leqq \tau \leqq 1,|h(z, \tau)|>0$ for $r_{1} \leqq z<a_{m_{1}}$ and such that (14) holds for all $r \geqq r_{1}$.

Similarly we choose $r_{2} \geqq r_{1}$ such that $-r_{2}$ is close enough to but $>-b_{m_{2}}$ for some $m_{2} \geqq 1$ with $|h(z, \tau)|>0$ for $-b_{m_{2}}<z \leqq-r_{2}$ and all $0 \leqq \tau \leqq 1$. 
Now we can define the curve $S$ as follows:

$$
\begin{aligned}
& S: z=r_{1} e^{i \theta} \quad \text { for }-\pi / 2 \leqq \theta \leqq \pi / 2, \quad z=r_{2} e^{i \theta} \quad \text { for } \pi / 2 \leqq \theta \leqq 3 \pi / 2 \text {, } \\
& z=r e^{i(\pi / 2)} \quad \text { for } r_{1} \leqq r \leqq r_{2}, \quad z=r e^{-i(\pi / 2)} \quad \text { for } r_{1} \leqq r \leqq r_{2} .
\end{aligned}
$$

Then $|h(z, \tau)|>0$ for $z \in S$ and $0 \leqq \tau \leqq 1$ and since $|h(z, \tau)|$ is continuous as a function of $(z, \tau) \in S \times[0,1]$ we actually have $|h(z, \tau)| \geqq c_{4}>0$ so that

$$
\frac{1}{2 \pi i} \int_{S}\left(\frac{h^{\prime}(z, \tau)}{h(z, \tau)}\right) d z
$$

is constant with respect to $\tau$ for $0 \leqq \tau \leqq 1$. Similarly as in Case I this integral is $\geqq l$ if $p$ is odd and $\geqq l+1$ if $p$ is even. Together with what has been proved earlier it follows that $g(z)$ has exactly $l$ zeros (exactly $l+1$ zeros) besides at least one zero between each pair $a_{n}, a_{n+1}$ and $-b_{n+1},-b_{n}$ and besides the trivial zeros at $a_{n}$ and $-b_{n}$ of multiplicities $\alpha_{n}-1$ and $\beta_{n}-1$ respectively if $p$ is odd (if $p$ is even).

Finally we want to end this section by giving some examples which show that the results of Theorem 1, Case II cannot be sharpened.

Let $\varphi(z)=e^{(\beta / 2) z^{2}} \cos z$ with real $\beta \neq 0$. Here $\rho=2$ and $p=1$. Furthermore let

$$
\gamma(z)=\alpha \varphi(z)+z \varphi^{\prime}(z)=e^{(\beta / 2) z^{2}}\left(\left(\alpha+\beta z^{2}\right) \cos z-z \sin z\right) \quad \text { with real } \alpha \neq 0 .
$$

We now apply Theorem 1, Case II, 1 to $g(z)=z \sin z-\left(\alpha+\beta z^{2}\right) \cos z$, where in the notation of Theorem $1 f(z)=\sin z, a=1, b=2, a_{1}=\pi,-b_{1}=-\pi$. From this theorem and from $g(-z)=g(z)$ follows that $g(z)$ has exactly one zero between each pair of consecutive positive and negative zeros of $\sin z$ and that $g(z)$ has exactly two complex zeros and exactly two real zeros in $(-\pi, \pi)$ if $\alpha, \beta$ have the same sign. In particular in $(-\pi / 2, \pi / 2) g(z)$ has no zero if $\alpha, \beta<0$ and exactly two zeros if $\alpha, \beta>0$.

We now apply Theorem 1, Case II,6 to $g(z)=-\left(\alpha+\beta z^{2}\right) \cos z+z \sin z$ where we consider $\cos z$ as $f(z), a=2, b=1$, and $a_{1}=\pi / 2,-b_{1}=-\pi / 2$. Then $g(z)$ also has exactly one zero between each pair of consecutive positive and negative zeros of $\cos z$. Besides these zeros $g(z)$ has exactly four zeros $(2$ complex and 2 in $(-\pi / 2, \pi / 2))$ if $\alpha, \beta>0$ and exactly two zeros $(2$ complex and no zeros in $(-\pi / 2, \pi / 2))$ if $\alpha, \beta<0$.

Therefore if $\alpha, \beta>0$ the upper bound, as given in Case II,6 is reached by $g(z)$ and if $\alpha, \beta<0$ it is not.

At the same time $\varphi(z)$ and $\gamma(z)$ are examples which show that Case II, 1 and Case II, 4 are best possible.

In Case II, 1 the condition $p \leqq \rho<p+1$ cannot be replaced by $p \leqq \rho \leqq p+1$, since in the above example $\rho=2, p=1$, but if $\alpha, \beta>0, \gamma(z)$ has exactly four zeros besides one between each pair of consecutive positive and negative zeros of $\varphi(z)$.

In addition this example shows that the upper bound, as given by Case II, 4 is reached. On the other hand if $\alpha, \beta<0$ this upper bound is not reached. 
Finally we want to show that Case II,3 and Case II,5 are best possible also. Let $\varphi(z)=e^{(\beta / 3) z^{3}} \cos z$ with real $\beta \neq 0$. Here $\rho=3, p=1$. Furthermore let $\gamma(z)=A(z) \varphi(z)$ $+B(z) \varphi^{\prime}(z)$, where $A(z)$ and $B(z)$ are real polynomials of exact degrees 3 and 1 respectively.

Then $\gamma(z)=e^{(\beta / 3) z^{3}} g(z)$ with $g(z)=\left(A(z)+\beta z^{2} B(z)\right) \cos z-B(z) \sin z$ and we also assume that $P(z)=A(z)+\beta z^{2} B(z)$ shall have degree 3 exactly. From Theorem 1 , Case II, 1 , applied to $g(z)$ with $f(z)=\sin z, a=1, b=3, a_{1}=\pi,-b_{1}=-\pi$, follows that $g(z)$ has exactly five zeros besides one between each pair of consecutive positive and negative zeros of $\sin z$ if $P(z)$ has its real zeros in $(-\pi, \pi)$. If we choose $B(z)$ and $P(z)$ such that $B(-\pi / 2)>0, B(\pi / 2)<0$ and $P(0)>0, P(\pi / 4)-B(\pi / 4)<0$, $P(-\pi / 4)+B(-\pi / 4)<0, P(-\pi)<0, P(\pi)>0$, then $g(z)$ has exactly four zeros in $(-\pi / 2, \pi / 2)$ and exactly five zeros in $(-\pi, \pi)$.

If we choose $B(z)$ and $P(z)$ such that $B(-\pi / 2)<0, B(\pi / 2)>0$ and $P(z) \equiv 0$ then $g(z)$ has exactly two zeros in $(-\pi / 2, \pi / 2)$.

Now Theorem 1, Case II,5 applied to $g(z)$ with $f(z)=\cos z, a=3, b=1, a_{1}=\pi / 2$, $-b_{1}=-\pi / 2$ shows that $g(z)$, for both choices of the pair $B(z), P(z)$ also has exactly one zero between each pair of consecutive positive and negative zeros of $\cos z$. In addition the upper bound, as given by Case II,5 is reached by $g(z)$ if $g(z)$ has four zeros in $(-\pi / 2, \pi / 2)$.

At the same time $\varphi(z)$ and $\gamma(z)$ are examples which show that the upper bound, as given by Case II,3 is reached in one case, while it is not reached in the other case.

In a similar way one can find examples which show that Cases I,2 and 3 are best possible by using $\cos \sqrt{ } z$ or $\sqrt{ } z \sin \sqrt{ } z$.

3. In the following let $f(z)=\sum_{n=0}^{\infty} a_{n} z^{n}$ denote a real, nonconstant, entire function of finite order $\rho$ and with $f(0) \neq 0$. Especially we want to restrict $f(z)$ to the following two types (for the definition of $p$ and $q$ see the beginning of $\S 2$ ):

$$
f(z)=c \prod_{n=1}^{\infty}\left(1-\frac{z}{c_{n}}\right) \text { with } \sum_{n=1}^{\infty}\left|c_{n}\right|^{-1}<\infty
$$

(i.e. either $\rho<1$ or $\rho=1$ and $p=q=0$ ),

$$
f(z)=c e^{b z} \prod_{n=1}^{\infty}\left(1-\frac{z}{c_{n}}\right) e^{z / c_{n}} \quad \text { with } \sum_{n=1}^{\infty}\left|c_{n}\right|^{-2}<\infty
$$

(i.e. either $\rho<2$ or $\rho=2$ and $q \leqq p=1$ ).

Here $b$ and $c$ shall be real and $c \neq 0$. Furthermore let $F(z)=\sum_{n=0}^{\infty} a_{n} G(n) z^{n}$, where $G(z)$ is a real entire function of the following two types:

(A) $G(z)=e^{\beta z} \prod_{n=1}^{\infty}\left(1+z / \alpha_{n}\right) e^{-z / \alpha_{n}}$, where each $\alpha_{n}>0, \beta$ real and $\sum_{n=1}^{\infty} \alpha_{n}^{-2}<\infty$. It is also allowed that $G(z)$ has finitely many (negative) zeros only.

(B) $G(z)=\left(z-\alpha_{1}\right) \cdots\left(z-\alpha_{m}\right)$, where $\alpha_{1}, \ldots, \alpha_{m}>0$. 
Then always $F(z)$ is a real entire function of order $\leqq \rho$. Having some information on the zeros of $f(z)$ we want to gain information on the zeros of $F(z)$. The following theorems, with the exception of Theorem 4, can be deduced from Theorem 1. The proof of Theorem 4 is independent of Theorem 1 .

THEOREM 2. Let $f(z)$ have infinitely many zeros but only $k$ zeros which are not $>0$.

(a) If $f(z)$ is of type (1) and $G(z)$ of type (A) then $F(z)$ has at most $k$ zeros which are not $>0$.

(b) If $f(z)$ is of type (1) and $G(z)$ of type (B) then $F(z)$ has at most $k+m$ zeros which are not $>0$.

If $f(z)$ is of type (2) and $G(z)$ of type (B) then $F(z)$ has at most $k+2 m$ zeros which are not $>0$. Besides $F(z)$ is of the same type as $f(z)$ and has infinitely many positive zeros. A corresponding result can be obtained if $f(z)$ has $k$ zeros which are not $<0$.

THEOREM 3. Let $f(z)$ be of type (2) with infinitely many real zeros of both signs and $2 k$ complex zeros.

(a) If $G(z)$ is of type (A) then $F(z)$ has at most $2 k$ complex zeros.

(b) If $G(z)$ is of type (B) then $F(z)$ has at most $2 k+2 m$ complex zeros and is of the same type as $f(z)$. Besides $F(z)$ has infinitely many real zeros of both signs.

THEOREM 4. Let $f(z)=\exp \left(a z^{2}\right) h(z)$ where $h(z)$ is of type (2) and $a \leqq 0$. Furthermore let $f(z)$ have real zeros only (or no zeros at all). If $G(z)$ is of type (A) then $F(z)$ has real zeros only.

THEOREM 5. Let $f(z)$ have infinitely many zeros but only $k$ zeros which are not $>0$. Furthermore assume that $G(z)$ is of type (B) having the positive zeros $\alpha_{1}, \ldots, \alpha_{m}$. With $f_{0}(z)=f(z)$ and $f_{j}(z)=-\alpha_{j} f_{j-1}(z)+z f_{j-1}^{\prime}(z)$ for $j=1, \ldots, m$ we assume that for each $j, f_{j}(z)$ has exactly one zero between each pair of consecutive positive zeros of $f_{j-1}(z)$ and no zero between $z=0$ and the smallest positive zero of $f_{j-1}(z)$.

If $f(z)$ is of type (1) then $F(z)$ has exactly $k+m$ zeros which are not $>0$. If $f(z)$ is of type (2) and $p=1$ then $F(z)$ has exactly $k+2 m$ zeros which are not $>0$.

In both cases $F(z)$ is of the same type as $f(z)$. A corresponding result holds if $f(z)$ has $k$ zeros which are not $<0$.

THEOREM 6. Let $f(z)$ be of type (2) with infinitely many real zeros of both signs and with exactly $2 k$ complex zeros. Furthermore assume that $G(z)$ is of type (B) having the positive zeros $\alpha_{1}, \ldots, \alpha_{m}$. With $f_{0}(z)=f(z)$ and $f_{j}(z)=-\alpha_{j} f_{j-1}(z)+z f_{j-1}^{\prime}(z)$ for $j=1, \ldots, m$ we assume that for each $j, f_{j}(z)$ has exactly one zero between each pair of consecutive positive and negative zeros of $f_{j-1}(z)$ and that $f_{j}(z)$ has no zero between the smallest positive and the smallest negative zero of $f_{j-1}(z)$.

Under these conditions $F(z)$ has exactly $2 k+2 m$ complex zeros. Besides $F(z)$ is of the same type as $f(z)$.

In order to prove Theorems 2-6 we need the following

LeMma 4. Let $g(z)=\alpha f(z)+z f^{\prime}(z)$ with real $\alpha \neq 0, a, b$ and $f(z)$ real. 
(1) If $f(z)=e^{b z} h(z)$ where $h(z)$ is of type (1), then $g(z)=e^{b z} h^{*}(z)$ where $h^{*}(z)$ is of type (1).

(2) If $f(z)=e^{a z^{2}} h(z)$ where $h(z)$ is of type (2), then $g(z)=e^{a z^{2}} h^{*}(z)$ where $h^{*}(z)$ is of type (2).

In both cases we assume that all but finitely many zeros of $h(z)$ are real. Then the same is true for $h^{*}(z)$. It is allowed that $h(z)$ has finitely many zeros only.

Proof. (1) We have $f^{\prime}(z) / f(z)=b+\sum_{n=1}^{\infty} 1 /\left(z-c_{n}\right)$ where all but finitely many $c_{n}$ are real. As $z$ tends to infinity along the imaginary axis $f^{\prime}(z) / f(z)$ tends to $b$. According to Hadamard's factorization theorem $g(z)$ is of the same form as $f(z)$. It remains to show that $g^{\prime}(z) / g(z)$ also tends to $b$ as $z$ tends to infinity along the imaginary axis. We have

$$
\frac{g^{\prime}}{g}=\frac{f^{\prime}}{f}+\frac{\left(f^{\prime} \mid f\right)+z\left(f^{\prime} \mid f\right)^{\prime}}{\alpha+z\left(f^{\prime} \mid f\right)}
$$

Here $z\left(f^{\prime} \mid f\right)^{\prime}$ tends to 0 as $z$ tends to infinity along the imaginary axis. In addition we have for $z=i y, y$ real:

$$
\left|\alpha+z \frac{f^{\prime}}{f}\right| \geqq|z|\left|b+\sum_{n=N+1}^{\infty} \frac{1}{z-c_{n}}\right|-\left|\sum_{n=1}^{N !} \frac{z}{z-c_{n}}\right|-|\alpha|
$$

where we assume that $c_{1}, \ldots, c_{N}$ consist of all complex zeros of $f(z)$. Here the first term on the right side is

$$
\geqq|z|\left|\operatorname{Im} \sum_{n=N+1}^{\infty} \frac{1}{z-c_{n}}\right|=y^{2} \sum_{n=N+1}^{\infty} \frac{1}{y^{2}+c_{n}^{2}},
$$

and this tends to infinity with $y$. Therefore $g^{\prime} / g$ also tends to $b$ as $y$ tends to infinity.

(2) Here we have

$$
\frac{1}{z} \frac{f^{\prime}}{f}=2 a+\frac{b}{z}+\frac{1}{z} \sum_{n=1}^{\infty}\left(\frac{1}{z-c_{n}}+\frac{1}{c_{n}}\right)
$$

where again all but finitely many $c_{n}$ are real. Therefore $f^{\prime} / z f$ tends to $2 a$ as $z$ tends to infinity along the imaginary axis. We divide equation (3) by $z$ and observe that now $\left(f^{\prime} \mid f\right)^{\prime}$ tends to $2 a$ as $z$ tends to infinity along the imaginary axis and that for the denominator $\alpha+z\left(f^{\prime} \mid f\right)$ we have for $a=0$ (the case $a \neq 0$ being trivial because of (3)):

$$
\left|\alpha+z \frac{f^{\prime}}{f}\right| \geqq|z|\left|b+\sum_{n=1}^{N} \frac{1}{c_{n}}+\sum_{n=N+1}^{\infty}\left(\frac{1}{z-c_{n}}+\frac{1}{c_{n}}\right)\right|-\left|\sum_{n=1}^{N} \frac{z}{z-c_{n}}\right|-|\alpha| .
$$

Here again we assume that $c_{1}, \ldots, c_{N}$ consists of all complex zeros of $f(z)$. Now if $z=i y$ is purely imaginary, the first term on the right side of (4) is

$$
\geqq|z|\left|\operatorname{Im} \sum_{n=N+1}^{\infty} \frac{1}{z-c_{n}}\right|
$$


which similarly as in the proof of (1) tends to infinity with $y$. This shows that $g^{\prime} / z g$ also tends to $2 a$ as $y$ tends to infinity.

Proof of Theorems 2 and 3. Let $\alpha$ be real and $\neq 0$. Observing that with $f(z)=$ $\sum_{n=0}^{\infty} a_{n} z^{n}$ one has $g(z)=\alpha f(z)+z f^{\prime}(z)=\sum_{n=0}^{\infty} a_{n}(\alpha+n) z^{n}$ we first prove the statement of the Theorem for the function $g(z)$.

From $g(z)=\alpha f(z)+z f^{\prime}(z)$ we see that always $g(z)$ has an odd (even) number of zeros between $z=0$ and the smallest positive and the smallest negative zero of $f(z)$ if $\alpha>0(\alpha<0)$.

To prove Theorem 2(a) we apply Theorem 1, Case I,1 to $g(z)$ with $\alpha>0$. Since $g(z)$ has at least one zero between $z=0$ and the smallest positive zero of $f(z)$ it follows that $g(z)$ has at most $k$ zeros which are not $>0$.

In order to prove part (b) of Theorem 2 we apply Theorem 1, Case I,1 to $g(z)$ again but now with $\alpha<0$, so that $g(z)$ has at most $k+1(k+2)$ zeros which are not $>0$ if $f(z)$ is of type (1) (type (2)).

In all cases $g(z)$ has infinitely many real zeros $>0$ and according to Lemma 4 $g(z)$ is of the same type as $f(z)$. Therefore $g(z)$ satisfies the same conditions of Theorem 2 as $f(z)$ does, the only difference being that in part (b) $k$ has to be replaced by $k+1(k+2)$ if $f(z)$ is of type (1) (type (2)).

In order to finish the proof of part (b) we apply the above arguments $m$ times where successively $\alpha$ is replaced by $-\alpha_{j}(j=1, \ldots, m)$. Then we obtain that

$$
\sum_{n=0}^{\infty} a_{n}\left(n-\alpha_{1}\right) \cdots\left(n-\alpha_{m}\right) z^{n} \quad \text { or } \quad F(z)=\sum_{n=0}^{\infty} a_{n} G(n) z^{n}
$$

has at most $k+m(k+2 m)$ zeros which are not $>0$ if $f(z)$ is of type (1) (type (2)).

To finish the proof of part (a) we repeat the above arguments with $\alpha_{1}, \ldots, \alpha_{l}>0$ and obtain that

$$
\sum_{n=0}^{\infty} a_{n}\left(\alpha_{1}+n\right) \cdots \cdot\left(\alpha_{l}+n\right) z^{n}
$$

for each $l$ has at most $k$ zeros which are not $>0$. This remains true if we divide this series by $\alpha_{1} \cdots \cdots \alpha_{l}$ and replace $z$ by $z \exp \left(\beta-\sum_{j=1}^{l} \alpha_{j}^{-1}\right)$ which gives the function $g_{l}(z)=\sum_{n=0}^{\infty} a_{n} G_{l}(n) z^{n}$ where $G_{l}(z)$ converges to $G(z)$ for each $z$ as $l$ tends to infinity. Since $\left|a_{n} G_{l}(n)\right|$ and $\left|a_{n} G(n)\right|$ are $\leqq\left|a_{n}\right| e^{\beta n}$ it follows that $g_{l}(z)$ converges, uniformly on compact domains, to the entire function $F(z)=\sum_{n=0}^{\infty} a_{n} G(n) z^{n}$, which according to a theorem of Hurwitz [6] still has at most $k$ zeros which are not $>0$.

Theorem 3 is proved in a similar way. The only difference is that now Theorem 1, Case II has to be applied instead of Case I.

Proof of Theorem 4. From the assumptions made about $f(z)$ it follows that

$$
\frac{f^{\prime}}{f}=2 a z+b+\sum_{n=1}^{\infty}\left(\frac{1}{z-c_{n}}+\frac{1}{c_{n}}\right)
$$


where all $c_{n}$ are real. Then we have $g(z)=\alpha f(z)+z f^{\prime}(z)=z f(z) \varphi(z)$ where

$$
\varphi(z)=\frac{\alpha}{z}+\frac{f^{\prime}}{f}=\frac{\alpha}{z}+2 a z+b+\sum_{n=1}^{\infty}\left(\frac{1}{z-c_{n}}+\frac{1}{c_{n}}\right) .
$$

Suppose that $z$ is not real, then

$$
\frac{\varphi(z)-\varphi(\bar{z})}{z-\bar{z}}=-\frac{\alpha}{|z|^{2}}+2 a-\sum_{n=1}^{\infty} \frac{1}{\left|z-c_{n}\right|^{2}}
$$

which is $<0$ if $a \leqq 0$ and $\alpha>0$. Consequently $g(z)$ does not have complex zeros if $\alpha>0$. According to Lemma $4 g(z)$ is of the same type as $f(z)$ (with $a \leqq 0$ !) and in order to finish the proof one only has to repeat the arguments of the proof of Theorem 2.

Theorems 5 and 6 finally are proved in the same way as Theorem 2(b). In addition one has to use the fact that Theorem 1 gives the exact number of zeros which, under the assumptions made in Theorems 5 and 6, cannot be $>0$ in Theorem 5 and which cannot be real in Theorem 6.

Next we want to apply the previous theorems in order to obtain Hurwitz's theorem on the zeros of Besselfunctions.

Let

$$
J_{v}(z)=\left(\frac{z}{2}\right)^{v} \sum_{n=0}^{\infty} \frac{(-1)^{n}(z / 2)^{2 n}}{n ! \Gamma(\nu+n+1)}
$$

denote the Besselfunction of order $\nu$, which is defined for all $z \neq 0$ and with $-\pi<\arg z<\pi$. We assume that $\nu$ is real and $\neq-1,-2, \ldots$ The zeros of $J_{v}(z)$ are determined by the zeros of the entire function

$$
\phi_{v}(z)=\sum_{n=0}^{\infty} \frac{(-1)^{n}(z / 2)^{2 n}}{n ! \Gamma(\nu+n+1)}
$$

This entire function is of order 1 as can be seen from the coefficients of the series. Therefore according to Hadamard's factorization theorem $p \leqq 1$ (in fact $p=1$, but this is not needed here). Furthermore $\phi_{\nu}(z)$ satisfies: $\phi_{v}(0) \neq 0$ and

(i) $\phi_{v}^{\prime}(z)=-(z / 2) \phi_{v+1}(z)$,

(ii) $2 \phi_{v}(z)=2(\nu+1) \phi_{v+1}(z)+z \phi_{v+1}^{\prime}(z)$,

(iii) $z \phi_{v}^{\prime \prime}(z)+(2 v+1) \phi_{v}^{\prime}(z)+z \phi_{v}(z)=0$.

Since $\varphi_{v}(z)=\phi_{v}(2 \sqrt{ } z)$ is an entire function of order $1 / 2$, again according to Hadamard's factorization theorem $\phi_{v}(z)$ has infinitely many zeros, which are simple because of (iii).

THEOREM 7 (HURWITZ's THEOREM). (a) $\phi_{v}(z)$ has real zeros only if $\nu>-1$.

(b) If $m$ is a natural number and $-(m+1)<\nu<-m$ then $\phi_{v}(z)$ has exactly $2 m$ complex zeros.

(c) If $m$ is odd $\phi_{v}(z)$ has exactly 2 purely imaginary zeros and if $m$ is even $\phi_{v}(z)$ has no purely imaginary zeros. 
Proof. (a) follows from Theorem 4 with $f(z)=e^{-(z / 2)^{2}},(\rho=2, p=0)$ and $G(z)=$ $1 / \Gamma(z / 2+\nu+1)$ which for $\nu>-1$ is of type (A).

(b) follows from Theorem 6 with

$$
f(z)=\sum_{n=0}^{\infty} \frac{(-1)^{n}(z / 2)^{2 n}}{n ! \Gamma(n+\nu+m+1)}, \quad(\rho=1, p \leqq 1)
$$

and $G(z)=(v+1+z / 2) \cdots(v+m+z / 2)$ which is of type (B). The remaining conditions made in 'Theorem 6 are satisfied here because of the properties (i) and (ii) with $\alpha_{j}=-2(\nu+j), j=1, \ldots, m$.

(c) follows from sign $\phi_{v}(0)=\operatorname{sign}(1 / \Gamma(\nu+1))$ and by using (i) and (ii) for purely imaginary $z$.

More generally we consider for arbitrary real $\alpha, \alpha J_{v}(z)+z J_{v}^{\prime}(z)=(z / 2)^{v} \psi_{v \alpha}(z)$ with $\psi_{v \alpha}(z)=(\alpha+\nu) \phi_{v}(z)+z \phi_{v}^{\prime}(z)$. If, for the moment, we put $g(z)=z^{\alpha} J_{v}(z)$, then

$$
g^{\prime}=z^{\alpha-1}\left(\alpha J_{v}+z J_{v}^{\prime}\right),
$$

and from the differential equation for $J_{v}(z)$ or $\phi_{v}(z)$ follows

$$
\left(z^{1-2 \alpha} g^{\prime}\right)^{\prime}=-z^{1-2 \alpha}\left(1+\left(\alpha^{2}-\nu^{2}\right) / z^{2}\right) g .
$$

If $|\nu| \leqq|\alpha|(5)$ shows that $g^{\prime}(z)$ or $\psi_{v \alpha}(z)$ has exactly one zero between each pair of consecutive positive zeros of $g(z)$ or $\phi_{v}(z)$ and that $g^{\prime}(z)$ has exactly one (has no) zero between $z=0$ and the smallest positive zero of $\phi_{v}(z)$ if $(\alpha+\nu)>0$ (if $\left.(\alpha+\nu)<0\right)$.

With these results Theorem 1, Case II, 1 gives the following

THEOREM 8. Let $\phi_{\nu}(z)$ have exactly $2 m$ complex zeros $(m \geqq 0)$. Then for any real $\alpha$ $\psi_{v \alpha}(z)$ has exactly $2 m+2$ zeros besides one zero between each pair of consecutive positive and negative zeros of $\phi_{v}(z)$.

Especially $\psi_{v \alpha}(z)$ has at most $2 m(2 m+2)$ complex zeros if $(\alpha+\nu)>0($ if $(\alpha+\nu)<0)$.

If furthermore $|\nu| \leqq|\alpha|$, then $\psi_{v \alpha}(z)$ has exactly $2 m$ (exactly $\left.2 m+2\right)$ complex zeros if $(\alpha+\nu)>0($ if $(\alpha+\nu)<0)$.

The following theorem again is an application of Theorem 1, Case II.

THEOREM 9. Let $\Pi(z)$ be a canonical product of genus $p$ with infinitely many real zeros of both signs, no complex zeros and no zero at $z=0$. Furthermore consider $g(z)=\alpha f(z)+z^{l} f^{\prime}(z)$, where $f(z)=z^{m} \Pi(z)$ with $m \geqq 0, \alpha$ real and $\geqq 0$ and $l$ odd.

(a) If $\alpha>0$ and $m \geqq 0$ then $g(z)$ has exactly $p+l-2(p+l-1)$ complex zeros when $p$ is odd (even).

(b) If $\alpha=0$ and $m>0$ then $g(z)$ has exactly $p-1(p)$ complex zeros when $p$ is odd (even).

(c) If $\alpha=m=0$ then $g(z)$ has no complex zeros.

REMARK. A similar result can be obtained if $\Pi(z)$ still has infinitely many real zeros but not infinitely many of both signs. One has to apply Theorem 1, Case I instead of Case II. 
Proof. $g(z)=z^{l+p} f(z) \varphi_{1}(z)$ if $p$ is even and $g(z)=z^{l+p-1} f(z) \varphi_{2}(z)$ if $p$ is odd. Here

$$
\varphi_{1}(z)=\frac{\alpha}{z^{l+p}}+\frac{m}{z^{p+1}}+\sum_{n=1}^{\infty} \frac{1}{a_{n}^{p}\left(z-a_{n}\right)} \quad \text { where } p \text { is even, }
$$

and

$$
\varphi_{2}(z)=\frac{\alpha}{z^{l+p-1}}+\frac{m}{z^{p}}+\sum_{n=1}^{\infty}\left(\frac{1}{a_{n}^{p-1}\left(z-a_{n}\right)}+\frac{1}{a_{n}^{p}}\right) \text { where } p \text { is odd. }
$$

Next we form the derivatives and see that $\varphi_{1}^{\prime}(z)$ and $\varphi_{2}^{\prime}(z)$ are $<0$ for real $z \neq 0$ and $\neq a_{n}$. Consequently $g(z)$ has exactly one zero between each pair of consecutive positive and negative zeros of $f(z)$. Furthermore if $\alpha>0$ and $m \geqq 0 g(z)$ has exactly one zero between $z=0$ and the smallest positive and negative zero of $f(z)$. In addition $g(z)$ has an $m$-fold zero at $z=0$ and thus according to Theorem 1, Case II we obtain (a).

Cases (b) and (c) are proved similarly.

Corollary. Let $\alpha>0$ and $l$ odd, then the functions $\alpha \sin z+z^{l} \cos z$ and $\alpha \cos z$ $-z^{l} \sin z$ have exactly $l-1$ complex zeros.

\section{REFERENCES}

1. E. Borel, Leçons sur les fonctions entières, 2nd ed., Gauthier-Villars, Paris, 1921.

2. E. Hille, Analytic function theory, Vol. II, Blaisdell, Waltham, Mass., 1962.

3. N. Obreschkoff, Verteilung und Berechnung der Nullstellen reeller Polynome, Deutscher Verlag der Wissenschaften, Berlin, 1963.

4. A. Peyerimhoff, On the zeros of power series, Michigan Math. J. 13 (1966), 193-214.

5. G. Pólya and G. Szegö, Aufgaben und Lehrsätze aus der Analysis, 2nd ed., Springer, Berlin, 1954.

6. E. C. Titchmarsh, The theory of functions, 2nd ed., Oxford Univ. Press, London, 1939.

MATHEMATISCHES INSTITUT DeR UNIVERSitÄt MARbURG, Marburg-Lahn, West Germany 